le portiQue $\begin{array}{ll}\text { Le Portique } \\ \text { Revue de philosophie et de sciences humaines }\end{array}$

33 | 2014

Straub !

\title{
Autour de La Grande Illusion de Jean Renoir
}

Jean-Marie Straub

\section{(2) OpenEdition}

Journals

Electronic version

URL: http://journals.openedition.org/leportique/2766

DOI: 10.4000/leportique.2766

ISSN: $1777-5280$

\section{Publisher}

Association "Les Amis du Portique"

Printed version

Date of publication: 1 May 2014

ISSN: 1283-8594

\section{Electronic reference}

Jean-Marie Straub, "Autour de La Grande Illusion de Jean Renoir", Le Portique [Online], 33 | 2014 , document 9. Online since 05 February 2016, connection on 12 April 2021. URL: http://

journals.openedition.org/leportique/2766 ; DOI: https://doi.org/10.4000/leportique.2766

This text was automatically generated on 12 April 2021

Tous droits réservés 


\title{
Autour de La Grande Illusion de Jean Renoir
}

\author{
Jean-Marie Straub
}

Jean-Marie Straub - Je ne vais pas vous faire de grand discours. Ce n'est pas moi qui ai décidé que je présenterai les films de la carte blanche ${ }^{1}$. On m'a dit: il faut faire une carte blanche. La carte blanche, je l'ai faite exprès limitée. J'ai même limité la limitation. Quand on veut se faire beau et qu'on fait des cartes blanches et qu'on est un peu connu, on essaie ou bien un éventail, ou bien on dit : je veux rien d'autre que tous les films de Stroheim par exemple. J'ai fait ça à Avignon, autour du festival de théâtre. Alors quand on a fait ça une fois, on n'a pas envie de recommencer. Et puis se faire beau avec trois films différents, c'est très difficile. Donc, j'ai choisi trois films qui sont apparentés. Bien que le film de Grémillon soit quelque chose d'un peu en dehors de tout. Renoir, pourquoi Renoir ? J'ai choisi des films où il y a des Allemands méchants. Je me suis dit : les Allemands méchants à Metz, ça va plaire. Et je voulais plaire avec une carte blanche. Voilà. Mais c'est la même histoire dans les deux films. Simplement, Renoir a fait La Grande Illusion je crois avant la guerre, et il a dit d'ailleurs : ça ne sert à rien, les films ne servent à rien, ça n'a pas empêché la guerre d'éclater. Ensuite, après la guerre, comme un de ses derniers films, il a fait Le Caporal épinglé, qui est une histoire qui n'est pas la même mais qui est très apparentée, très semblable, très similaire. Mais qui regarde déjà l'avenir d'après-guerre plus directement.

2 Pourquoi ces deux films de Renoir ? Parce que, Renoir, c'est le cinéaste que je préfère. J'ai mis dix ans à le préférer à tous les autres. Bon. Il m'agaçait beaucoup au début, je le trouvais, comme John Ford, un peu sentimental. Et il a fallu que je prenne un tout petit peu de bouteille pour m'y intéresser. Ensuite, j'ai découvert que c'était le cinéaste le plus concret, le seul vraiment concret. Il y a des cinéastes plus grands, il y a Stroheim, il y a même Dreyer, il y a même Feuillade. Il y a Mizoguchi, il y a Fritz Lang. Je ne veux donc pas dire que c'est le plus grand cinéaste, mais c'est le plus particulier, celui qui a le mieux compris ce que c'est que le cinéma. C'est-à-dire un petit va et vient entre le théâtre et la vie. Voilà. L'abstraction et la réalité. Voilà. Quant à La Grande Illusion, c'était le film de Renoir que j'aimais le moins parmi tous les films de Renoir. Et j'ai eu 
envie de le revoir ce soir. Je ne l'ai pas vu depuis 15 ans. Surtout qu'entre temps, je suis allé tourner un petit film au Mont Sainte-Odile qui est le pendant du HautKoenigsbourg dans les Vosges. C'est au Haut-Koenigsbourg que Renoir a tourné $\mathrm{La}$ Grande illusion. Moi, le Haut-Koenigsbourg, je ne l'avais jamais vu. Je suis allé le voir pour la première fois il y a 6 mois. Et j'ai envie de le voir sur l'écran. Et je vous souhaite une bonne soirée. Et après, vous me direz tout ce que vous avez envie de vous dire, ce que vous avez envie de me dire...

\section{Après la projection}

Spectateur - Ce film a été tourné en 1937, à ma connaissance. Est-ce que Renoir l'a tourné en pensant à ce qui se passait en Allemagne à cette époque-là ? La guerre d'Espagne ? Est-ce qu'il l'a tourné en ayant l'impression que la Der des der, il allait y en avoir une suivante? Puisqu'après, il a dit : finalement, ce film n'a pas empêché qu'il y ait encore une guerre entre la France et l'Allemagne. c'était 15 ans avant. Parce que maintenant j'ai 117 ans ! Ça fait beaucoup d'années. Ce qui fait la force d'un film comme ça c'est la variété des tons. Ça, ça n'existe que chez Renoir. L'éventail. C'est un éventail extrêmement ouvert, qui va du grotesque au sublime. Surtout ça n'est jamais naturaliste. Chaque fois qu'on côtoie le naturalisme, il y a le théâtre qui intervient. Prenez l'exemple de Rosenthal qui se retourne au moment de quitter la pièce. Tout d'un coup, il se retourne et il part comme ça. Ça, ça n'existe que chez Renoir. C'est le contraire de toutes les séries télé qui veulent faire semblant d'être vraies. C'est du faux-semblant, c'est du toc. Ici, c'est le contraire. Donc, l'éventail des sentiments est ouvert.

11 Spectateur - Dans ce film, il y a ce sentiment de classe, qu'on trouve souvent chez Renoir, c'est très marquant. Les personnages sont définis par leur classe sociale.

12 J.-M. S. - Renoir a fait un film qui s'appelle La Marseillaise, qui a été produit par le Front populaire. années 30, c'était un cinéma d'acteurs. On voit Carette, Dalio, Gaston Modot .. 
18 J.-M. S. - Renoir a toujours utilisé les acteurs en tant qu'amis, pas en tant que clowns professionnels. Il les a pris à l'envers, souvent. C'est ça qui fait que ça tient debout. Je dirais pas à rebrousse-poil, mais pourquoi pas ! Vous voulez en venir où?

Spectateur - Nulle part. Je suis admiratif de ces acteurs de second rôle qui étaient formidables, dans les années 1930.

J.-M. S. - Lui-même a joué des rôles dans La Bête humaine et dans d'autres films. D'ailleurs, il a toujours été considéré comme un mauvais acteur. Chaque fois qu'il apparaissait, les assistants se mettaient à ricaner. J'entends : son apparition en tant qu'acteur. Justement parce qu'il était juste. Ce n'est pas un naturaliste. Ce qu'il fait avec tous ces acteurs de l'époque, qui étaient ses amis ou pas, qui étaient connus ou à la mode ou pas, c'est exactement pareil. C'est ça qui fait que Renoir, c'est Renoir.

21 Spectateur - Vous avez dit que Renoir était un cinéaste concret. Que c'est pour ça que vous l'aimiez parmi tous les autres cinéastes. Vous pouvez nous expliquer ce que vous entendez par l'aspect concret des films de Renoir?

J.-M. S. - C'est ce qu'on vient de dire là. Ce n'est pas très mystérieux. Ce qu'on vient de dire là. Il est concret avec la réalité, il est concret avec ses personnages, il est concret avec le son, etc. C'est un ensemble qui fait que... C'est un concret qui dialogue avec l'abstraction. C'est un concret qui doit sa force aussi à l'abstraction.

Bernard Muscat (Ciné Art) - L'économie de ses films : il arrivait à trouver des sous facilement pour faire ses films ? Comment ça se passait ? Qui il employait?

24 J.-M. S. - Renoir était dans... Il n'y a jamais eu d'industrie du cinéma français, mais il y avait un système commercial du cinéma français. Il était dedans. Ses films ont eu beaucoup moins de succès que ceux de Duvivier ou Carné, etc., mais ses films circulaient. Il y en a qui n'ont pas marché. Celui-ci n'a pas marché du tout. La Règle du jeu n'a pas marché. Une partie de campagne n'a pas marché. Madame Bovary n'a pas marché. Mais néanmoins, ça rapportait ce que ça avait coûté. Il disait dans la première interview qu'il a faite en rentrant en France avec François Truffaut, Rivette et je ne sais plus qui, il disait : oui, moi, j'étais à une course de taureau en Espagne, avec des amis, j'y comprenais rien, mais j'ai compris qu'il fallait comprendre, qu'il fallait savoir pour comprendre. Le torero fait un petit mouvement comme ceci à gauche, tout le monde se mettait à applaudir, un petit mouvement comme ceci à droite, tout le monde se mettait à hurler. Il dit : vous voyez, le cinéma, c'est pareil, c'est fait pour les aficionados. Et puis il enchaînait en disant : on croit que le cinéma, c'est fait pour les 6000 personnes du Gaumont Palace. Non, c'est fait pour 3 parmi ces 6000 personnes ! Voilà ce qu'il disait. Et pourtant, c'était un homme qui croyait au grand public et au cinéma commercial. C'est ça aussi la contradiction. Ou pour employer un grand mot, la dialectique. Il disait : on fait des films aussi seulement pour des amis. Il y en a un que j'aimais beaucoup, que j'estimais énormément, il a vu Madame Bovary avant qu'on le mutile d'une heure, en 36. Il l'a vu et ça lui plaisait beaucoup. Et ça m'a fait grand plaisir, c'est très important. On lui dit: qui c'est, ce monsieur que vous estimez tant et qui a vu Madame Bovary avant qu'on en coupe une heure ? Il a dit : il s'appelait Berthold Brecht, il était de passage à Paris. Il y a une belle petite histoire. Il n'y en a pas beaucoup parce que les gens sont vaniteux. Mais l'estime entre deux cinéastes, elle existe. Moi, quand j'ai l'impression que je vois un film qui est beaucoup plus fort que ce que j'aurais pu faire, je suis un peu jaloux, mais en même temps, je suis tout le contraire de jaloux, je suis enfin heureux. John Ford, très peu de temps avant de mourir, on lui a dit : alors, quel est le cinéaste 
que vous préférez ? Il a dit : oh ben, Renoir, Renoir, Renoir. D'ailleurs, il y a un film de John Ford où il reprend Dalio, des gens de LA règle du jeu. Il y a même une phrase en français pour qu'on comprenne bien. C'est la situation de Dalio qui présente son orgue mécanique dans La Règle du jeu. C'est un peu décalé. Le film s'appelle La Taverne de l'Irlandais. Alors il disait : oui, Renoir. Mais quel Renoir? Le journaliste s'attendait à ce qu'il lui dise : La Règle du jeu. Mais il a dit : non, tous les Renoir. Tous les Renoir. Mais ça, c'est très rare. C'est très rare et très beau.

\section{NOTES}

1. . Durant cette Rétrospective J.-M. S. a choisi de donner à voir différents films : de Jean Renoir, Jean Grémillon et des Marx Brothers. 